\title{
Non-Integrable Galactic Dynamics
}

\section{David Merritt}

Rutgers University, New Brunswick, NJ USA

\section{INTRODUCTION}

Galaxies have traditionally been viewed as integrable or nearly integrable systems, in which the majority of stellar orbits are regular, respecting as many integrals of motion as there are degrees of freedom. Three arguments have commonly been cited in support of this view. First, many reasonable potentials contain only modest numbers of stochastic orbits . This is always true for the potentials of rotationally symmetric models, and there is even a class of non-axisymmetric potentials for which the motion is globally integrable, including the famous "perfect ellipsoid" (Kuzmin 1973; de Zeeuw \& Lynden-Bell 1985). Second, stochastic orbits often behave in ways that are very similar to regular orbits over astronomically interesting time scales. Therefore (it is argued) one need not make a sharp distinction between regular and stochastic orbits when constructing an equilibrium model. Third, following the successful construction by Schwarzschild (1979, 1982) of self-consistent triaxial equilibria, it has generally been assumed that the regular orbits which are confined to narrow regions of phase space and thus have definite shapes - are the fundamental building blocks of real galaxies.

Schwarzschild's discovery that many orbits in non-axisymmetric potentials are effectively regular came as a surprise, since triaxial potentials admit only one classical integral of the motion, the energy. In fact a modest fraction of the orbits in Schwarzschild's models were subsequently shown to be stochastic (Merritt 1980; Goodman \& Schwarzschild 1981), though only weakly. But it was clear early on that certain modifications of Schwarzschild's potential could lead to a much larger fraction of chaotic orbits. For instance, Gerhard \& Binney (1985) showed that the addition of a central density cusp or "black hole" (i.e. point mass) to an otherwise integrable triaxial model would render most of the centerfilling, box orbits unstable, due to deflections that occur when a trajectory comes close to the center. This insight was followed by the discovery (Crane et al. 1993; Ferrarese et al. 1994) that stellar spheroids generically contain power-law cusps in the luminosity density

\footnotetext{
${ }^{1}$ The terms "stochastic" and "chaotic" will be used interchangeably here.
} 
rather than constant-density cores. Evidence for central supermassive black holes also gradually accumulated (Kormendy \& Richstone 1995). It is now believed - not only that black holes are universal components of galactic nuclei - but that their masses are predictable with high precision given the global properties of their host spheroids (Ferrarese \& Merritt 2000; Merritt \& Ferrarese 2001). Thus it is no longer possible to discuss galaxy dynamics in terms of idealized models like Schwarzschild's with finite central densities.

Non-integrability has two important consequences. First, some orbits in non-integrable potentials respect fewer isolating integrals than there are degrees of freedom. Such orbits are chaotic and behave in ways that are very different from regular orbits: they are exponentially unstable to small perturbations, and occupy a phase-space region of larger dimensionality than the invariant tori of regular orbits. The time-averaged shape of a chaotic orbit is similar to that of an equipotential surface and hence such orbits are much less "useful" than regular orbits for reinforcing the shape of the galaxy's figure. Second, while regular orbits generally still exist in potentials that are not globally integrable, they are strongly influenced by resonances between the frequencies of motion in different directions. These resonances are present even in globally integrable potentials but have no effect on the structure of phase space; in non-integrable potentials, however, the resonances divide up phase space into alternating regions of regular and chaotic motion, with the lowest-order resonances "capturing" the largest parts of phase space. Most regular orbits in non-integrable potentials can be associated with a definite resonance and have a shape that reflects the order of the resonance.

This article reviews the following topics: (1) Torus construction, a set of techniques for characterizing regular motion in non-integrable potentials and for detecting departures from integrability; (2) resonances and their effect on the structure of orbits; (3) the orbital content of triaxial potentials with central point masses; (4) mixing, the process by which the phase-space density of stellar systems approaches a steady state; and (5) the relation between chaos in the gravitational $N$-body problem and chaos in smooth potentials.

\section{TORUS CONSTRUCTION}

In systems with a single degree of freedom, constancy of the energy allows the momentum variable $p$ to be written in terms of the coordinate variable $q$ as $H(p, q)=E$, and the dependence of both variables on time follows immediately from Hamilton's equations. In general systems with $N \geq 2$ degrees of freedom (DOF), such a solution is generally not possible unless the Hamilton-Jacobi equation is separable, in which case the separation constants are isolating integrals of the motion. An isolating integral is a conserved quantity that in some transformed coordinate system makes $\partial H / \partial p_{i}=f\left(q_{i}\right)$, thus allowing the motion in $q_{i}$ to be reduced to quadratures. Each isolating integral restricts the dimensionality of the phase space region accessible to an orbit by one; if there are $N$ such integrals, the orbit moves in a phase space of dimension $2 N-N=N$, and the motion is regular. The $N$-dimensional phase space region to which a regular orbit is confined is topologically a torus (Figure 1). Orbits in time-independent potentials may be either regular or chaotic; chaotic orbits respect a smaller number of integrals than $N$ - typically

only the energy integral $E$. Although chaotic orbits are not confined to tori, numerical integrations suggest that many chaotic trajectories are effectively regular, remaining con- 
fined for long periods of time to regions of phase space much more restricted than the full energy hypersurface.

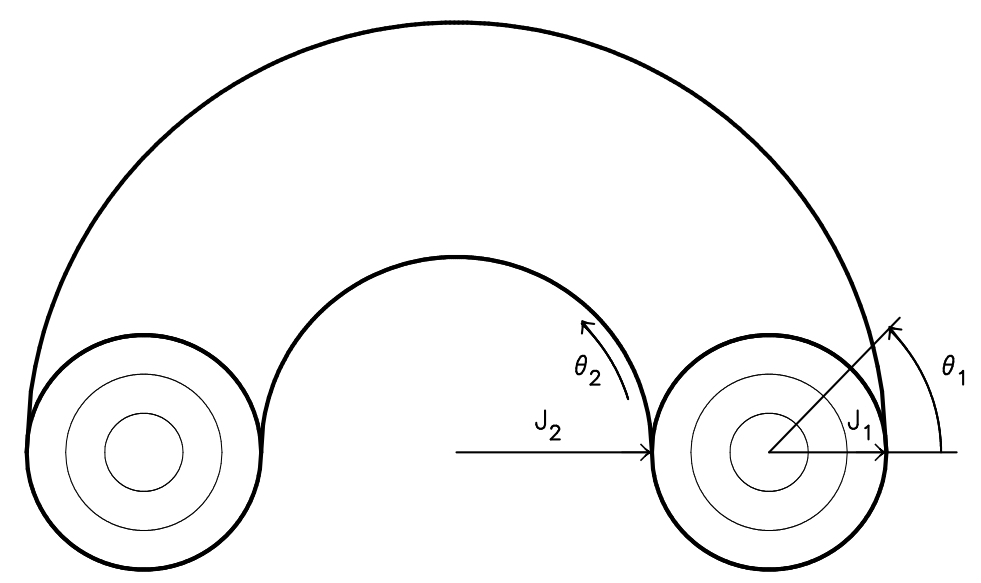

Figure 1. Invariant torus defining the motion of a regular orbit in a two-dimensional potential. The torus is determined by the values of the actions $J_{1}$ and $J_{2}$; the position of the trajectory on the torus is defined by the angles $\theta_{1}$ and $\theta_{2}$, which increase linearly with time, $\theta_{i}=\omega_{i} t+\theta_{i}^{0}$.

The most compact representation of a regular orbit is in terms of the coordinates on the torus (Figure 1 ) - the action-angle variables $(\mathbf{J}, \theta)$. The process of determining the $\operatorname{map}(\mathbf{x}, \mathbf{v}) \rightarrow(\mathbf{J}, \theta)$ is referred to as torus construction. There are a number of contexts in which it is useful to know the $(\mathbf{J}, \theta)$. One example is the response of orbits to slow changes in the potential, which leave the actions $(\mathbf{J})$ unchanged. Another is the behavior of weakly chaotic orbits, which may be approximated as regular orbits that slowly diffuse from one torus to another. A third example is galaxy modeling, where regular orbits are most efficiently represented and stored via the coordinates that define their tori.

Two general approaches to torus construction have been developed. Trajectoryfollowing algorithms are based on the quasi-periodicity of regular motion: Fourier decomposition of the trajectory yields the fundamental frequencies on the torus as well as the spectral amplitudes, which allow immediate construction of the map $\theta \rightarrow \mathbf{x}$ in the form of a Fourier series. Iterative approaches begin from some initial guess for $\mathbf{x}(\theta)$, which is then refined via Hamilton's equations with the requirement that the $\theta_{i}$ increase linearly with time. The two approaches are often complementary, as discussed below.

\subsection{Regular Motion}

In certain special potentials, every orbit is regular; examples are the Kepler and Stäckel potentials. Motion in such globally-integrable potentials can be expressed most simply by finding a canonical transformation to coordinates $(\mathbf{p}, \mathbf{q})$ for which the Hamiltonian is independent of $\mathbf{q}, H=H(\mathbf{p})$; among all such coordinates, one particularly simple choice is the action-angle variables $\left(J_{i}, \theta_{i}\right)$, in terms of which the equations of motion are

$$
\begin{aligned}
& J_{i}=\text { constant } \\
& \theta_{i}=\omega_{i} t+\theta_{i}^{0}, \quad \omega_{i}=\frac{\partial H}{\partial J_{i}}, \quad i=1, \ldots, N
\end{aligned}
$$


(Landau \& Lifshitz 1976; Goldstein 1980). The trajectory $\mathbf{x}(\mathbf{J}, \theta)$ is periodic in each of the angle variables $\theta_{i}$, which may be restricted to the range $0<\theta_{i} \leq 2 \pi$. The $J_{i}$ define the cross-sectional areas of the torus while the $\theta_{i}$ define the position on the torus (Figure 1). These tori are sometimes called "invariant" since a phase point that lies on a torus at any time will remain on it forever.

Most potentials are not globally integrable, but regular orbits may still exist; indeed these are the orbits for which torus construction machinery is designed. One expects that for a regular orbit in a non-integrable potential, a canonical transformation $(\mathbf{x}, \mathbf{v}) \rightarrow(\mathbf{J}, \theta)$ can be found such that

$$
\dot{J}_{i}=0, \quad \dot{\theta}_{i}=\omega_{i}, \quad i=1, \ldots, N .
$$

However there is no guarantee that the full Hamiltonian will be expressible as a continuous function of the $J_{i}$ as in globally integrable potentials. In general, the map $(\mathbf{x}, \mathbf{v}) \rightarrow(\mathbf{J}, \theta)$ $\mathrm{w}$ ill be different for each orbit and will not exist for those trajectories that do not respect $N$ isolating integrals.

The uniform translation of a regular orbit on its torus implies that the motion in any canonical coordinates $(\mathbf{x}, \mathbf{v})$ is quasi-periodic:

$$
\begin{aligned}
& \mathbf{x}(t)=\sum_{k} \mathbf{X}_{k}(\mathbf{J}) \exp \left[i\left(l_{k} \omega_{1}+m_{k} \omega_{2}+n_{k} \omega_{3}\right) t\right], \\
& \mathbf{v}(t)=\sum_{k} \mathbf{V}_{k}(\mathbf{J}) \exp \left[i\left(l_{k} \omega_{1}+m_{k} \omega_{2}+n_{k} \omega_{3}\right) t\right],
\end{aligned}
$$

with $\left(l_{k}, m_{k}, n_{k}\right)$ integers. The Fourier transform of $\mathbf{x}(t)$ or $\mathbf{v}(t)$ will therefore consist of a set of spikes at discrete frequencies $\omega_{k}=l_{k} \omega_{1}+m_{k} \omega_{2}+n_{k} \omega_{3}$ that are linear combinations of the $N$ fundamental frequencies $\omega_{i}$, with spectral amplitudes $\mathbf{X}_{k}(\mathbf{J})$ and $\mathbf{V}_{k}(\mathbf{J})$.

\subsection{Trajectory-Following Approaches}

The most straightforward, and probably the most robust, approach to torus construction is via Fourier analysis of the numerically-integrated trajectories (Percival 1974; Boozer 1982; Binney \& Spergel 1982, 1984; Kuo-Petravic et al. 1983; Eaker et al. 1984; Martens \& Ezra 1985). The Fourier decomposition of a quasiperiodic orbit (equation 3) yields a discrete frequency spectrum. The precise form of this spectrum depends on the coordinates in which the orbit is integrated, but certain of its properties are invariant, including the $N$ fundamental frequencies $\omega_{i}$ from which every line is made up, $\omega_{k}=l_{k} \omega_{1}+m_{k} \omega_{2}+n_{k} \omega_{3}$. Typically the strongest line in a spectrum lies at one of the fundamental frequencies; once the $\omega_{i}$ have been identified, the integer vectors $\left(l_{k}, m_{k}, n_{k}\right)$ corresponding to every line $\omega_{k}$ are uniquely defined, to within computational uncertainties. Approximations to the actions may then be computed using Percival's (1974) formulae; e.g. the action associated with $\theta_{1}$ in a 3 DOF system is

$$
J_{1}=\sum_{k} l_{k}\left(l_{k} \omega_{1}+m_{k} \omega_{2}+n_{k} \omega_{3}\right)\left|\mathbf{X}_{k}\right|^{2}
$$

Finally, the maps $(\theta \rightarrow \mathbf{x})$ are obtained by making the substitution $\omega_{i} t \rightarrow \theta_{i}$ in the spectrum, e.g.

$$
x(t)=\sum_{k} X_{k}(J) \exp \left[i\left(l_{k} \omega_{1}+m_{k} \omega_{2}+n_{k} \omega_{3}\right) t\right]
$$




$$
\begin{aligned}
& =\sum_{k} X_{k}(J) \exp \left[i\left(l_{k} \theta_{1}+m_{k} \theta_{2}+n_{k} \theta_{3}\right)\right] \\
& =x\left(\theta_{1}, \theta_{2}, \theta_{3}\right) .
\end{aligned}
$$

Trajectory-following algorithms are easily automated; for instance, integer programming may be used to recover the vectors $\left(l_{k}, m_{k}, n_{k}\right)$ (Valluri \& Merritt 1998).

Binney \& Spergel (1982) pioneered the use of trajectory-following algorithms for galactic potentials. They integrated orbits for a time $T$ and computed discrete Fourier transforms, yielding spectra in which each frequency spike was represented by a peak with finite width $\sim \pi / T$ centered on $\omega_{k}$. They then fitted these peaks to the expected functional form $X_{k} \sin \left[\left(\omega-\omega_{k}\right) T\right] /\left(\omega-\omega_{k}\right)$ using a least-squares algorithm. They were able to recover the fundamental frequencies in a 2 DOF potential with an accuracy of $\sim 0.1 \%$ after $\sim 25$ orbital periods. Binney \& Spergel (1984) used equation (4) to construct the "action map" for orbits in a principal plane of the triaxial logarithmic potential. Carpintero \& Aguilar (1998) have applied similar algorithms to motion in 2- and 3 DOF potentials.

The accuracy of Fourier transform methods can be greatly improved by multiplying the time series with a windowing function before transforming. The result is a reduction

in the amplitude of the side lobes of each frequency peak at the expense of a broadening of the peaks; the amplitude measurements are then effectively decoupled from any errors in the determination of the frequencies. Laskar $(1988,1990)$ developed this idea into a set of tools, the "numerical analysis of fundamental frequencies" (NAFF), which he applied to the analysis of weakly chaotic motion in the solar system. Laskar's algorithm recovers the fundamental frequencies with an error that falls off as $T^{-4}$ (Laskar 1996), compared with $\sim T^{-1}$ in algorithms like Binney \& Spergel's (1982). Even for modest integration times of $\sim 10^{2}$ orbital periods, the NAFF algorithm is able to recover fundamental frequencies with accuracies of $\sim 10^{-8}$ or better in many potentials. The result is a very precise representation of the torus (Figure 2).

Since Fourier techniques focus on the frequency domain, they are particularly well suited to identifying regions of phase space associated with resonances. Resonant tori are places where perturbation expansions of integrable systems break down, due to the "problem of small denominators". In perturbed (non-integrable) potentials, one expects stable resonant tori to generate regions of regular motion and unstable resonant tori to give rise to chaotic regions. Algorithms like NAFF allow one to construct a "frequency map" of the phase space: a plot of the ratios of the fundamental frequencies $\left(\omega_{1} / \omega_{3}, \omega_{2} / \omega_{3}\right)$ for a large a set of orbits selected from a uniform grid in initial condition space. Resonances appear on the frequency map as lines, either densely filled lines in the case of stable resonances, or gaps in the case of unstable resonances; the frequency map is effectively a representation of the Arnold web (Laskar 1993). Resonances are discussed in more detail in $\S 3$.

\subsection{Iterative Approaches}

Iterative approaches to torus construction consist of finding successively better approximations to the map $\theta \rightarrow \mathbf{x}$ given some initial guess $\mathbf{x}(\theta)$; canonical perturbation theory is a special case, and in fact iterative schemes often reduce to perturbative methods in appropriate limits. Iterative algorithms were first developed in the context of semi-classical 

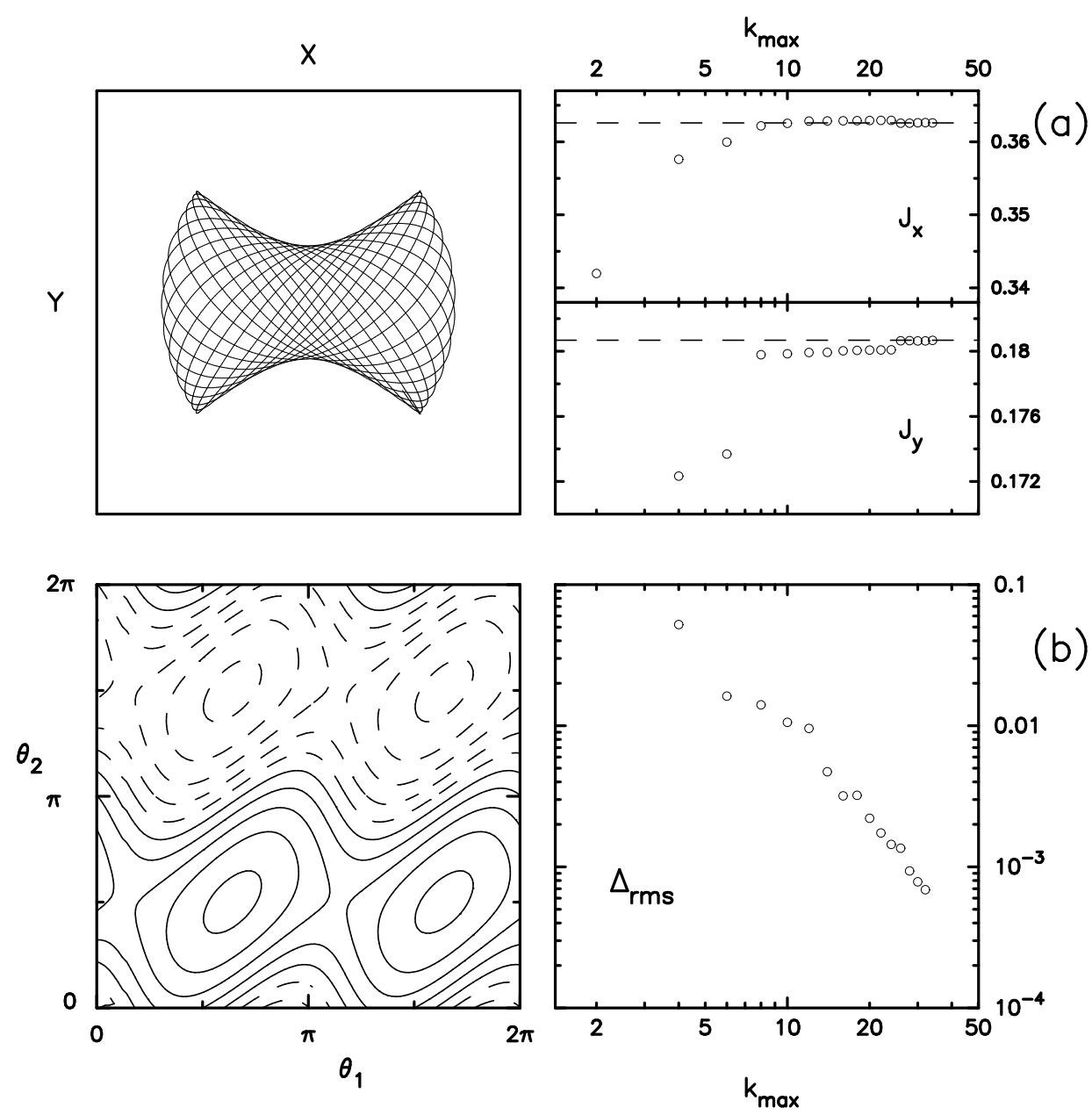

Figure 2. Construction of a 2 DOF, box-orbit torus in a Stäckel potential using the NAFF trajectory-following algorithm. (a) The orbit and its actions, computed using equation (4) with $k_{\max }$ terms. Dashed lines show the exact $J_{i}$. (b) The map $y\left(\theta_{1}, \theta_{2}\right)$; dashed contours correspond to negative values of $y . \Delta\left(k_{\max }\right)$ is the RMS error in the reconstructed map, calculated using an equation similar to (5).

quantization for computing energy levels of bound molecular systems, and they are still best suited to assigning energies to actions, $H(\mathbf{J})$. Most of the other quantities of interest to galactic dynamicists - e.g. the fundamental frequencies $\omega_{i}$ - are not easily recovered using these algorithms. Iterative schemes also tend to be numerically unstable unless the initial guess is close to the true solution. On the other hand, iterative algorithms can be more efficient than trajectory-following algorithms for orbits that are near (but not exactly on) resonances.

Ratcliff, Chang \& Schwarzschild (1984) pioneered iterative schemes in galactic dynamics. They noted that the equations of motion of a 2 DOF regular orbit,

$$
\ddot{x}=-\frac{\partial \Phi}{\partial x}, \quad \ddot{y}=-\frac{\partial \Phi}{\partial y}
$$

can be written in the form

$$
\left(\omega_{1} \frac{\partial}{\partial \theta_{1}}+\omega_{2} \frac{\partial}{\partial \theta_{2}}\right)^{2} x=-\frac{\partial \Phi}{\partial x}
$$




$$
\left(\omega_{1} \frac{\partial}{\partial \theta_{1}}+\omega_{2} \frac{\partial}{\partial \theta_{2}}\right)^{2} y=-\frac{\partial \Phi}{\partial y}
$$

If one specifies $\omega_{1}$ and $\omega_{2}$ and treats $\partial \Phi / \partial x$ and $\partial \Phi / \partial y$ as functions of the $\theta_{i}$, equations (可) can be viewed as nonlinear differential equations for $x\left(\theta_{1}, \theta_{2}\right)$ and $y\left(\theta_{1}, \theta_{2}\right)$. Ratcliff et al. expressed the coordinates as Fourier series in the angle variables,

$$
\mathbf{x}(\theta)=\sum_{n} \mathbf{X}_{n} e^{i n \cdot \theta}
$$

Substituting (8) into (17) gives

$$
\sum_{n}(\mathbf{n} \cdot \omega)^{2} \mathbf{X}_{n} e^{i n \cdot \theta}=\nabla \Phi
$$

where the right hand side is again understood to be a function of the angles. Ratcliff et al. truncated the Fourier series after a finite number of terms and required equations (9) to be satisfied on a grid of points around the torus. They then solved for the $\mathbf{X}_{n}$ by iterating from an initial guess. Convergence was found to be possible if the initial guess was close to the exact solution. A similar algorithm was developed for recovering tori in the case that the actions, rather than the frequencies, are specified a priori. Guerra \& Ratcliff (1990) applied these algorithms to motion in the plane of rotation of a nonaxisymmetric potential.

Another iterative approach to torus construction was developed by Chapman, Garrett \& Miller (1976) in the context of semiclassical quantum theory. One begins by dividing the Hamiltonian $H$ into separable and non-separable parts $H_{0}$ and $H_{1}$, then seeks a generating function $S$ that maps the known tori of $H_{0}$ into tori of $H$. For a generating function of the $F_{2}$-type (Goldstein 1980), one has

$$
\mathbf{J}\left(\theta, \mathbf{J}^{\prime}\right)=\frac{\partial S}{\partial \theta}, \quad \theta^{\prime}\left(\theta, \mathbf{J}^{\prime}\right)=\frac{\partial S}{\partial \mathbf{J}^{\prime}}
$$

where $(\mathbf{J}, \theta)$ and $\left(\mathbf{J}^{\prime}, \theta^{\prime}\right)$ are the action-angle variables of $H_{0}$ and $H$ respectively. The generator $S$ is determined, for a specified $\mathbf{J}^{\prime}$, by substituting the first of equations (10) into the Hamiltonian and requiring the result to be independent of $\theta$. One then arrives at $H\left(\mathbf{J}^{\prime}\right)$. Chapman et al. showed that a sufficiently general form for $S$ is

$$
S\left(\theta, \mathbf{J}^{\prime}\right)=\theta \cdot \mathbf{J}^{\prime}-i \sum_{n \neq 0} S_{n}\left(\mathbf{J}^{\prime}\right) e^{i n \cdot \theta}
$$

where the first term is the identity transformation, and they evaluated a number of iterative schemes for finding the $S_{n}$. One such scheme was found to recover the results of first-order perturbation theory after a single iteration. McGill \& Binney (1990) applied the Chapman et al. algorithm to 2 DOF motion in the axisymmetric logarithmic potential.

The generating function approach is not naturally suited to deriving the other quantities of interest to galactic dynamicists. For instance, equation (10) gives $\theta^{\prime}(\theta)$ as a derivative of $S$, but since $S$ must be computed separately for every $\mathbf{J}^{\prime}$ its derivative is likely to be ill-conditioned. Binney \& Kumar (1993) and Kaasalainen \& Binney (1994a) discussed two schemes for finding $\theta^{\prime}(\theta)$; the first requires the solution of a formally infinite 
set of equations, while the latter requires multiple integrations of the equations of motion for each torus - effectively a trajectory-following scheme.

Warnock (1991) presented a hybrid scheme in which the generating function $S$ was derived by numerically integrating an orbit from appropriate initial conditions, transforming the coordinates to $(\mathbf{J}, \theta)$ of $H_{0}$ and interpolating $\mathbf{J}$ on a regular grid in $\theta$. The values of the $S_{n}$ then follow from the first equation of (10) after a discrete Fourier transform. Kaasalainen \& Binney (1994b) found that Warnock's scheme could be used to substantially refine the solutions found via their iterative algorithm. Another hybrid scheme was discussed by Reiman \& Pomphrey (1991).

Having computed the energy on a grid of $\mathbf{J}^{\prime}$ values, one can interpolate to obtain the full Hamiltonian $H\left(\mathbf{J}^{\prime}\right)$. If the system is not in fact completely integrable, this $H$ may be rigorously interpreted as smooth approximation to the true $H$ (Warnock \& Ruth 1991, 1992) and can be taken as the starting point for secular perturbation theory. Kaasalainen (1994) developed this idea and showed how to recover accurate surfaces of section in the neighborhood of low-order resonances in the planar logarithmic potential.

Percival (1977) described a variational principle for constructing tori. His technique has apparently not yet been implemented in the context of galactic dynamics.

\subsection{Chaotic Motion}

Torus-construction machinery may be applied to orbits that are approximately, but not precisely, regular (Laskar 1993). The frequency spectrum of a weakly chaotic orbit will typically be close to that of a regular orbit, with most of the lines well approximated as linear combinations of three "fundamental frequencies" $\omega_{i}$. However these frequencies will change with time as the orbit migrates from one "torus" to another. The diffusion rate can be measured via quantities like $\delta \omega=\left|\omega_{1}-\omega_{1}^{\prime}\right|$, the change in a "fundamental frequency" over two consecutive integration intervals. Papaphilippou \& Laskar (1996, 1998), Valluri \& Merritt (1998) and Wachlin \& Ferraz-Mello (1998) used this technique to study chaos and diffusion in triaxial galactic potentials.

Measuring chaos via quantities like $\delta \omega$ has a number of advantages over the traditional technique based on computation of the Liapunov exponents (Lichtenberg \& Lieberman 1992). $\delta \omega$ can be accurately determined after just a few tens of orbital periods, whereas determination of the Liapunov exponents may require much longer integrations. The Liapunov exponents measure only the rate of growth of infinitesimal perturbations around the trajectory, while $\delta \omega$ measures the finite "movement" of the trajectory in action-angle

space, a more physically interesting measure of chaos. It is possible for orbits to be extremely unstable in the sense of having large Liapunov exponents, but to behave nearly regularly in the sense of having small $\delta \omega$; an example is presented in $\S 6$.

\section{RESONANCES}

The character of a regular orbit depends critically on whether the fundamental frequencies $\omega_{i}$ are independent, or whether they satisfy one or more nontrivial linear relations of the 
form

$$
\sum_{i=1}^{N} m_{i} \omega_{i}=0
$$

with $N$ the number of degrees of freedom and $m_{i}$ integers, not all of which are zero. Generally there exists no relation like equation (12); the frequencies are incommensurate, and the trajectory fills its invariant torus uniformly and densely in a time-averaged sense. When one or more resonance relations are satisfied, however, the trajectory is restricted to a phase-space region of lower dimensionality than $N$.
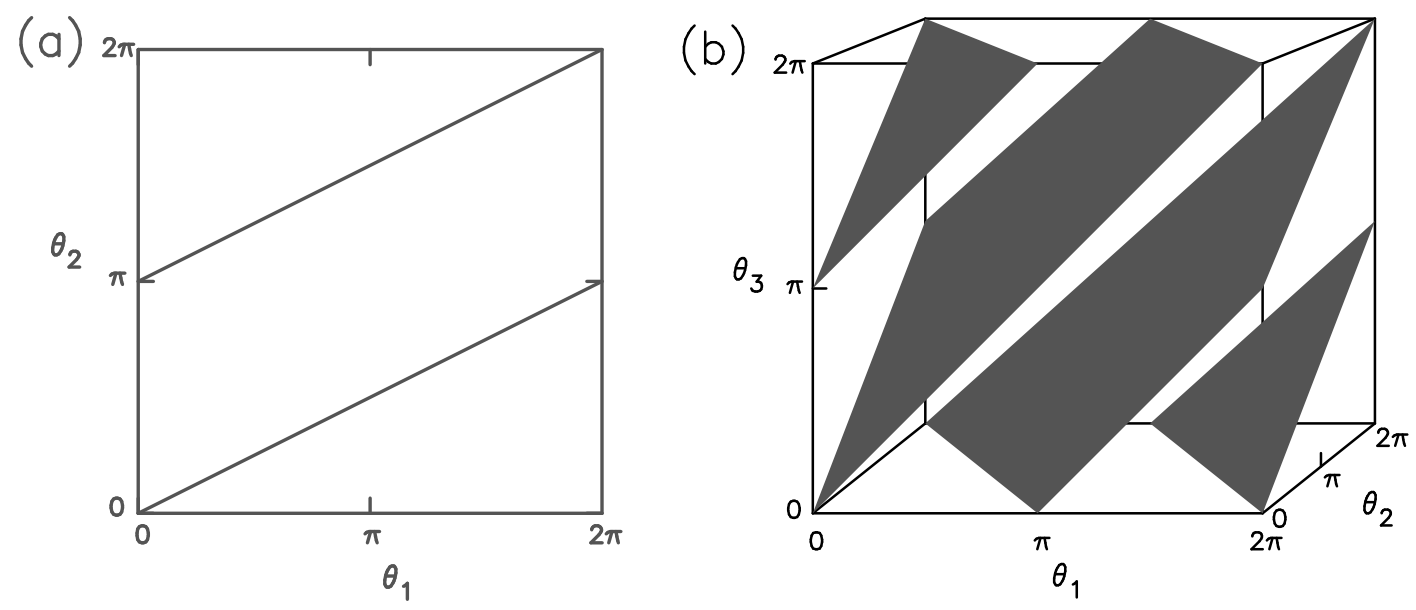

Figure 3. Resonant tori. (a) A two-dimensional torus, shown here as a square with identified edges. The plotted trajectory satisfies a $2: 1$ resonance between the fundamental frequencies, $\omega_{1}-2 \omega_{2}=0$ (e.g. a "banana"). (b) A three-dimensional torus, shown here as a cube with identified sides. The shaded region is covered densely by a resonant trajectory for which $2 \omega_{1}+\omega_{2}-2 \omega_{3}=0$. This trajectory is not closed, but it is restricted by the resonance condition to a two-dimensional subset of the torus. The orbit in configuration space is thin.

In the case of a two-dimensional regular orbit, the angle variables are

$$
\theta_{1}=\omega_{1} t+\theta_{10}, \quad \theta_{2}=\omega_{2} t+\theta_{20}
$$

which define the surface of a torus (Fig. 1). Because of the quasi-periodicity of the orbit, its torus can be mapped onto a square in the $\left(\theta_{1}, \theta_{2}\right)$-plane, with each side ranging from 0 to $2 \pi$ (Figure $3 \mathrm{a}$ ); the top and bottom of the square are identified with each other, as are the left and right sides. In the general case, the frequencies $\omega_{1}$ and $\omega_{2}$ are incommensurate and the trajectory densely covers the entire $\left(\theta_{1}, \theta_{2}\right)$-plane after an infinite time. However if the ratio $\omega_{1} / \omega_{2}=\left|m_{2} / m_{1}\right|$ is a rational number, i.e. if $m_{1}$ and $m_{2}$ are integers, the orbit closes on itself after $\left|m_{2}\right|$ revolutions in $\theta_{1}$ and $\left|m_{1}\right|$ revolutions in $\theta_{2}$ and fills only a one-dimensional subset of its torus (e.g. Arnold 1963, p. 164). Its dimensionality in configuration space is also one - the orbit is closed. Such an orbit has a single fundamental frequency $\omega_{0}=\omega_{1} / m_{2}=\omega_{2} / m_{1}=2 \pi / T$, with $T$ the orbital period; after an elapsed time $T$, the trajectory returns to its starting point in phase space. Examples of resonant orbits in two-dimensional galactic potentials are the "boxlets" (Miralda-Escudé \& Schwarzschild 1989). 
In the case of a three-dimensional regular orbit, the angle variables are

$$
\theta_{1}=\omega_{1} t+\theta_{10}, \quad \theta_{2}=\omega_{2} t+\theta_{20}, \quad \theta_{3}=\omega_{3} t+\theta_{30}
$$

The orbit may now be mapped into a cube whose axes are identified with the $\theta_{i}$ (Figure $3 \mathrm{~b})$. If the $\omega_{i}$ are incommensurate, this cube will be densely filled after a long time. However if a single condition of the form

$$
m_{1} \omega_{1}+m_{2} \omega_{2}+m_{3} \omega_{3}=0
$$

is satisfied with integer $m_{i}$, the motion is restricted for all time to a two-dimensional subset of its torus. Such an orbit is not closed; instead, as suggested by Figure 3b, it is thin, confined to a sheet or membrane in configuration space, which it fills densely after infinite time.

Just as in the two-dimensional case, the condition (15) may be used to reduce the number of independent frequencies by one. Defining the two "base" frequencies $\omega_{0}^{(1)}, \omega_{0}^{(2)}$ as

$$
\omega_{0}^{(1)}=\omega_{3} / m_{1}, \quad \omega_{0}^{(2)}=\omega_{2} / m_{1}
$$

we may write

$$
\begin{aligned}
& \omega_{1}=-m_{3} \omega_{0}^{(1)}-m_{2} \omega_{0}^{(2)} \\
& \omega_{2}=m_{1} \omega_{0}^{(2)} \\
& \omega_{3}=m_{1} \omega_{0}^{(1)}
\end{aligned}
$$

Since the motion is quasi-periodic, i.e.

$$
\mathbf{x}(t)=\sum_{k} \mathbf{X}_{k} \exp i\left(l_{k} \omega_{1}+m_{k} \omega_{2}+n_{k} \omega_{3}\right) t
$$

with $\left(l_{k}, m_{k}, n_{k}\right)$ integers, it will remain quasi-periodic when expressed in terms of the two base frequencies:

$$
\begin{aligned}
& \mathbf{x}(t)=\sum_{k} \mathbf{X}_{k} \exp i\left[\left(-l_{k} m_{3}+n_{k} m_{1}\right) \omega_{0}^{(1)}+\left(-l_{k} m_{2}+m_{k} m_{1}\right) \omega_{0}^{(2)}\right] t \\
& =\sum_{k} \mathbf{X}_{k} \exp i\left(l_{k}{ }^{\prime} \omega_{0}^{(1)}+m_{k}{ }^{\prime} \omega_{0}^{(2)}\right) t \\
& =\sum_{k} \mathbf{X}_{k} \exp i\left(l_{k}^{\prime} \theta^{(1)}+m_{k}^{\prime} \theta^{(2)}\right) \\
& l_{k}{ }^{\prime}=-l_{k} m_{3}+n_{k} m_{1}, \quad m_{k}{ }^{\prime}=-l_{k} m_{2}+m_{k} m_{1}, \\
& \theta^{(1)}=\omega_{0}^{(1)} t, \quad \theta^{(2)}=\omega_{0}^{(2)} t \text {. }
\end{aligned}
$$

A Fourier transform of the motion will therefore consist of a set of spikes whose locations can be expressed as linear combinations of just two frequencies. Equation (19) is a parametric expression for the Cartesian coordinates in terms of the angles on the 2-torus, i.e. it is a reconstruction of the (reduced) torus. A number of examples of resonant box orbits reconstructed in this way are illustrated in Figures 4 and 5. 
Certain special orbits may satisfy two independent resonance relations simultaneously. In this case we can write:

$$
\begin{gathered}
m_{1} \omega_{1}+m_{2} \omega_{2}+m_{3} \omega_{3}=0, \\
n_{1} \omega_{1}+n_{2} \omega_{2}+n_{3} \omega_{3}=0,
\end{gathered}
$$

and each frequency $\omega_{i}$ may be expressed as a rational fraction of any other:

$$
\frac{\omega_{1}}{\omega_{3}}=\frac{m_{2} n_{3}-m_{3} n_{2}}{m_{1} n_{2}-m_{2} n_{1}}=\frac{l_{1}}{l_{3}}, \quad \frac{\omega_{2}}{\omega_{3}}=\frac{m_{3} n_{1}-m_{1} n_{3}}{m_{1} n_{2}-m_{2} n_{1}}=\frac{l_{2}}{l_{3}},
$$

with $\left(l_{1}, l_{2}, l_{3}\right)$ integers. The motion is therefore periodic with a single base frequency $\omega_{0}=\omega_{1} / l_{1}=\omega_{2} / l_{2}=\omega_{3} / l_{3}$ and the trajectory is closed - the orbit is a three-dimensional, closed curve. In a system with $N$ degrees of freedom, $N-1$ such conditions are required for closure; only in the 2DOF case does a single resonance condition imply closure.

Following Poincaré (1892), it has commonly been assumed that closed orbits are the fundamental "building blocks" of phase space. However in three-dimensional potentials, one expects thin orbits to be more common than closed ones, in the sense that orbits satisfying one resonance condition are more likely than orbits satisfying two. Hence one expects that most regular orbits will be associated with families whose parent is a thin orbit. Numerical integrations of orbits in realistic nonaxisymmetric potentials suggest that this is in fact the case: the majority of regular orbits have most of their "power" in frequencies that lie close to linear combinations of two fundamental frequencies (thin orbit) rather than one frequency (closed orbit) (Merritt \& Valluri 1999; Figure 7).

\section{ORBITAL STRUCTURE OF TRIAXIAL POTEN- TIALS WITH CENTRAL SINGULARITIES}

Non-integrability is likely to be a generic feature of galactic potentials, for two reasons. First, galaxies are often observed to be non-axisymmetric, either due to the presence of imbedded sub-systems like bars, or because the stellar distribution is globally triaxial. Observational evidence for global triaxiality in elliptical galaxies is not particularly strong; few ellipticals exhibit significant minor-axis rotation (Franx, Illingworth \& de Zeeuw 1991), and detailed modelling of a handful of nearby ellipticals suggests that their kinematics can often be very well reproduced by assuming axisymmetry (e.g. van der Marel et al. 1998). However, at least some elliptical galaxies and bulges exhibit clear kinematical signatures of non-axisymmetry (s.g. Schechter \& Gunn 1979; Franx, Illingworth \& Heckman 1989), and the observed distribution of Hubble types is likewise inconsistent with the assumption that all ellipticals are precisely axisymmetric (Tremblay \& Merritt 1995, 1996; Ryden 1996). Mergers between disk galaxies also produce generically triaxial systems (Barnes 1996), and departures from axisymmetry (possibly transient) are widely argued to be necessary for the rapid growth of nuclear black holes during the quasar epoch (Shlosman, Begelman \& Frank 1990), for the fueling of starburst galaxies (Sanders \& Mirabel 1996), and for the large radio luminosities of some ellipticals (Bicknell et al. 1997). These arguments suggest that most elliptical galaxies or bulges may have been triaxial at an earlier epoch, and perhaps that triaxiality is a recurrent phenomenon induced by mergers or other interactions. 
The second feature of galactic potentials that is conducive to non-integrability is the apparently universal presence at the centers of stellar spheroids of high stellar densities and supermassive black holes. Low-luminosity ellipticals and bulges have stellar luminosity profiles that diverge as unbroken power laws at small radii, $\rho \sim r^{-\gamma}$, with $\gamma \approx 2$. Brighter galaxies also exhibit power laws in the space density of stars, but with shallower slopes, $\gamma \lesssim 1$; seen in projection, these weaker cusps appear as cores (Kormendy 1985). The gravitational force in an $r^{-2}$ density cusp diverges as $r^{-1}$, not steep enough to produce large-angle deflections in the motion of stars that pass near the center. However galaxies also contain supermassive black holes, with masses that correlate astonishingly well with the velocity dispersion of the stars (Ferrarese \& Merritt 2000); the ratio of black hole mass to spheroid mass is $\sim 0.0015$ with small scatter (Merritt \& Ferrarese 2001). The combination of non-axisymmetry in the potential with a steep central force gradient is conducive to non-integrability and chaos, since many orbits in non-axisymmetric potentials pass near the center where they undergo strong gravitational deflections (Gerhard \& Binney 1985).

In a triaxial potential containing a central point mass, the phase space divides naturally into three regions depending on energy, i.e. on distance from the center (Figure 6). In the innermost region, where the enclosed mass in stars is less than the mass of the black hole, the potential is dominated by the central singularity and the motion is essentially regular. The gravitaional force from the stars acts as a small perturbation causing the nearly-Keplerian orbits around the black hole to slowly precess. The two major orbit families in this region are the tube orbits, high angular momentum orbits that avoid the center; and the pyramid orbits, Keplerian ellipses that precess in two orthogonal planes parallel to the short axis of the figure (Sridhar \& Touma 1999; Sambhus \& Sridhar 2000; Poon \& Merritt 2001). Pyramid orbits are similar to the classical box orbits of integrable triaxial potentials except that their elongation is counter to that of the triaxial figure, making them less useful for self-consistently reconstructing a galaxy's shape.

At intermediate radii, the black hole acts as a scattering center rendering almost all of the center-filling or box orbits stochastic. (Tube orbits persist at these and higher energies and remain mostly regular.) This "zone of chaos" extends from a few times $r_{g}$, the radius where the black hole dominates the gravitational force, out to a radius where the enclosed stellar mass is roughly $10^{2}$ times the mass of the black hole. The transition to chaos at $r \gtrsim r_{g}$ is very rapid and occurs at lower energies in more elongated potentials (Poon \& Merritt 2001).

If the black hole mass exceeds $\sim 10^{-2}$ times the mass of the stellar spheroid, as it may do in a few galaxies (Merritt \& Ferrarese 2001), the chaotic zone will include essentially the entire potential outside of $\sim r_{g}$. However if $M_{\bullet} \approx 10^{-3} M_{\text {gal }}$, as in the majority of galaxies, there exists a third, outermost region where the phase space is a complex mixture of chaotic and regular trajectories, including resonant box orbits like those in Figures 4 and 5 that remain stable by avoiding the center (Carpintero \& Aguilar 1998; Papaphillipou \& Laskar 1998; Valluri \& Merritt 1998; Wachlin \& Ferraz-Mello 1998). Figure 7 illustrates the complexity of box-orbit phase space at large energies in two triaxial potentials: one with a weak density cusp and the other with a central point mass.

Non-integrable potentials often exhibit a transition to global stochasticity as the magnitude of some perturbation parameter is increased. The results summarized above suggest that there are two such perturbation parameters associated with motion in triaxial 
galaxies containing central black holes. In a triaxial galaxy with a given $M_{\bullet}$, the motion of center-filling orbits undergoes a sudden transition to stochasticity as the energy is increased; the critical value is the energy at which the gravitational force from the stars is of order the force from the black hole. If one imagines increasing $M_{\bullet}$ in an otherwise fixed, triaxial potential, the zone of chaos that extends outward from this radius will eventually encompass the entire potential; this occurs when the second "perturbation parameter," $M_{\bullet} / M_{\text {gal }}$, exceeds $\sim 10^{-2}$. Thus at intermediate radii, in the "zone of chaos," and perhaps throughout an elliptical galaxy containing a central black hole, triaxiality should be difficult to maintain.

\section{MIXING AND COLLISIONLESS RELAXATION}

Stochastic motion introduces a new time scale into galactic dynamics, the mixing time. Mixing is the process by which a non-uniform distribution of particles in phase space relaxes to a uniform distribution, at least in a coarse-grained sense. A weak sort of mixing, phase mixing, occurs even in integrable potentials, as particles on adjacent tori gradually move apart (Lynden-Bell 1967; Figure 8a). Phase mixing is responsible for the fact that the coarse-grained phase space density in relaxed integrable systems is nearly constant around tori. A stronger sort of mixing takes place in chaotic systems. Chaotic motion is essentially random in the sense that the likelihood of finding a particle anywhere in the stochastic region tends toward a constant value after a sufficiently long time. An initially compact group of stars should therefore spread out until it covers the accessible phase space region uniformly in a coarse-grained sense (Kandrup \& Mahon 1994; Figure 8b). This "chaotic mixing" is irreversible in the sense that an infinitely fine tuning of velocities would be required in order to undo its effects. It also occurs on a characteristic time scale, the Liapunov time associated with exponential divergence of nearby trajectories. Phase mixing, by contrast, has no associated time scale; its rate depends on the range of frequencies associated with orbits in the region of interest, and this rate tends to zero in the case of a set of trajectories drawn from a single invariant torus - a set of points on the torus translates, unchanged, around the torus.

Figure 9 shows examples of chaotic mixing in a triaxial potential with a central point mass. Ensembles of orbits were started at rest on an equipotential surface and integrated in tandem for several crossing times. The central point had a mass $M_{\bullet}=0.03$ in units of the galaxy mass. The first ensemble (a) was begun on an equipotential surface enclosing a mass $\sim 3$ times that of the central point; for ensembles (b) and (c) these ratios were $\sim 7$ and $\sim 17$ respectively - all within the "zone of chaos." Mixing occurs very rapidly in these ensembles. At the lowest energy (ensemble a), the linear extent of the points in configuration space roughly doubles every crossing time until $T \approx 4$, when the volume defined by the equipotential surface appears to be nearly filled. At the highest energy (ensemble c), mixing is slower but substantial changes still take place in a few crossing times. The final distribution of points at this energy still shows some structure, reminiscent of a box orbit.

The irreversibility of mixing flows like the ones illustrated in Figure 9 implies a reduction in the effective number of orbits: all the stochastic trajectories at a given energy are gradually replaced by a single invariant ensemble, whose shape is typically not well 
matched to that of the galaxy (Merritt \& Fridman 1996). If time scales for chaotic mixing are comparable to galaxy lifetimes, this reduction might be expected to encourage a galaxy to evolve away from a triaxial shape toward a more axisymmetric one, in which most of the orbits are tubes that avoid the destabilizing center. Such evolution has in fact been observed in $N$-body simulations of the response of a triaxial galaxy to the growth of a central black hole. Merritt \& Quinlan (1998) found that a triaxial galaxy evolves to axisymmetry in little more than the local crossing time at each radius when the black hole mass exceeds $\sim 2.5 \%$ of the total galaxy mass. This is about an order of magnitude larger than the typical black hole mass ratio in real galaxies (Merritt \& Ferrarese 2001),

but Merritt \& Quinlan observed more gradual evolution even when the mass ratio was 10 times smaller, at a rate that would imply substantial shape changes over a galaxy lifetime. These simulations suggest an explanation for the generally low level of triaxiality observed in real galaxies (Bak \& Statler 2000).

\section{CHAOS IN COLLISIONAL SYSTEMS}

The discussion presented so far has assumed that galaxy potentials are smooth, or "collisionless." In fact, the gravitational force on a star in a galaxy can be broken up into two components: a rapidly varying component that arises from the discrete distribution of stars, and a smoothly varying component that arises from the large-scale matter distribution. The effects of the discrete component of the force relative to the smooth component are usually assumed to scale as $\sim \ln N / N$, the ratio of dynamical to two-body relaxation times. For galaxies, which have $N \sim 10^{11}$, collisional effects should therefore be unimportant.

If this were the case, it should be possible to show that the $N$-body trajectories go over, in the limit of large $N$, to the orbits in the corresponding smoothed-out potential i.e., that the equations of motion of the $N$-body problem tend to the characteristics of the collisionless Boltzmann equation as $N \rightarrow \infty$. However this has never been demonstrated, and in fact there is an important sense in which the equations of motion in an $N$-body system do not tend toward the trajectories of the corresponding smooth potential in the limit of large $N$.

This surprising statement is justified in Figure 10, which shows the results of testparticle integrations in a potential consisting of $N$ fixed point masses distributed randomly and uniformly within a triaxial ellipsoid. The mass of each of the $N$ points is $m=1 / N$, so that the total mass and mean density of the ellipsoid remain constant as $N$ is varied. In the limit $N \rightarrow \infty$, one might expect the equations of motion to approach those of a 3d harmonic oscillator, since the potential of a uniform ellipsoid is quadratic in the coordinates, $\Phi=\Phi_{0} \sum_{i} A_{i} x_{i}^{2}$ (Chandrasekhar 1969). However the upper left-hand panel shows that the Liapunov exponents $\sigma$ of orbits in the $N$-body potential do not tend to zero with increasing $N$. Instead, the instability time scale appears to $\mathrm{r}$ each a roughly constant value (expressed as a fraction of the crossing time $T_{c r}$ ) for $N \gtrsim 10^{3}$. Furthermore the instability time scale is very short, a fraction of the crossing time!

The generic instability of the $N$-body problem was first noted by Miller (1964), who calculated the time evolution of the separation between two $N$-body systems with slightly 
different initial conditions. He defined this separation as

$$
\Delta(t)=\left[\sum\left(\mathbf{x}_{2}-\mathbf{x}_{\mathbf{1}}\right)^{2}+\sum\left(\mathbf{v}_{2}-\mathbf{v}_{\mathbf{1}}\right)^{2}\right]^{1 / 2}
$$

with $\mathbf{x}_{1}$ and $\mathbf{x}_{2}$ the $N$ configuration-space coordinates in $N$-body systems 1 and 2 and $\mathbf{v}_{1}$ and $\mathbf{v}_{2}$ the velocities; the summations extend over all the particles. Miller found, for $4 \leq N \leq 32$, that $\Delta$ grew roughly exponentially with a characteristic time scale that was a fraction of the crossing time, as in the fixed $N$-body problem of Figure 10.

What are the physical implications of this generic instability? Several suggestions have been made. Gurzadyan \& Saviddy (1986), who first investigated the large- $N$ dependence of the instability using an idealized model, suggested that the exponential divergence implies chaotic mixing on a similar time scale, and hence that stellar systems should relax much more rapidly than implied by the standard Chandrasekhar formula. Heggie (1991) disagreed, but suggested that the use of smooth potentials for approximating galaxies would need to be abandoned, at least for studies of orbital instability. Kandrup (1998) suggested that - while individual orbits may always be exponentially unstable - ensembles of $N$-body systems might behave, on average, as if the potential were smooth.

Figure 10 suggests an even stronger way in which the motion goes over to that of the collisionless problem as $N \rightarrow \infty$. The open circles in the upper left-hand panel of that figure show a second measure of the orbital evolution: the rms variation, over 20 orbital periods, of the action $J_{x}$ for each ensemble of orbits. Contrary to the behavior of the Liapunov exponents, the average changes in the actions tend uniformly to zero as $N$ is increased - in other words, the orbits approach more and more closely, in their macroscopic behavior, to that of integrable orbits even though they remain locally unstable (as measured by the Liapunov exponents) to a degree that is nearly independent of $N$. Plots of the trajectories of some typical orbits (lower left panel) confirm this interpretation. These results suggest the way in which trajectories in the $N$-body problem tend toward those in the corresponding smooth potential: as $N$ is increased, orbits are confined more and more strongly to narrow regions of phase space around the invariant tori of the smooth potential.

It is remarkable that orbits can be extremely unstable locally, as measured by their Liapunov exponents, and yet behave macroscopically in a way that is essentially identical to that of regular orbits. Apparently, the exponential growth of perturbations must saturate at some finite amplitude, and this saturation amplitude must be a decreasing function of $N$. The lower right-hand panel of Figure 10 verifies this conjecture for a few pairs of orbits with nearly identical initial conditions. The early divergence takes place at a rate that is independent of $N$, but for large $N$, the separation saturates at a value that is much smaller than the size of the system. These pairs of orbits act as if they are confined to the same, restricted region of phase space; saturation occurs when the separation between them is of order the width of this region. The fact that the exponential divergence saturates sooner for larger $N$ suggests that the width of the confining regions decreases with increasing $N$.

These results suggest that collisional relaxation in stellar systems is intimately connected with the evolution of orbits under conditions of weak chaos, i.e., with Arnold diffusion. This connection would be a fruitful topic for future study.

Some of the work presented here was first published in collaboration with M. Valluri. 
I am grateful for her permission to reproduce the work here. The preparation of this review was supported by NSF grant AST-0071099 and by NASA grants NAG 5-2803 and NAG 5-9046.

\section{References}

Arnold, V. I. 1963, Russian Mathematical Surveys, 18, 85

Bak, J. \& Statler, T. 2000, AJ, 120, 110

Barnes, J. 1996, in The Formation of Galaxies, Proceedings of the V Canary Islands Winter School of Astrophysics, ed. C. Muñoz-Tuñón (Cambridge: Cambridge University Press), 399

Bicknell, G. V., Koekemoer, A., Dopita, M. A. \& O'Dea, C. P. 1997, in The Second Stromlo Symposium: The Nature of Elliptical Galaxies, A.S.P. Conf. Ser. Vol. 116, eds. M. Arnaboldi, G. S. Da Costa \& P. Saha) (Provo: ASP), 432

Binney, J. \& Kumar, S. 1993, MNRAS, 261, 584

Binney, J. \& Spergel, D. 1982, ApJ, 252, 308

Binney, J. \& Spergel, D. 1984, MNRAS, 206, 159

Boozer, A. H. 1982, Phys. Fluids, 25, 520

Carpintero, D. D. \& Aguilar, L. A. 1998, MNRAS, 298, 1

Chandrasekhar, S. 1969, Ellipsoidal Figures of Equilibrium (New York: Dover)

Chapman, S., Garrett, B. C. \& Miller, W. H. 1976, J. Chem. Phys., 64, 502

Crane, P. et al. 1993, AJ, 106, 1371

de Zeeuw, P. T. \& Lynden-Bell, D. 1985, MNRAS, 215, 713

Eaker, C. W., Schatz, G. C., De Leon, N. \& Heller, E. J. 1984, J. Chem. Phys., 81, 5913

Ferrarese, L. et al. 1994, $A J, \mathbf{1 0 8}, 1598$

Ferrarese, L. \& Merritt, D. 2000, ApJ, 539, L9

Franx, M., Illingworth, G. D. \& de Zeeuw, P. T. 1991, ApJ, 383, 112

Franx, M., Illingworth, G. D. \& Heckman, T. M. 1989, ApJ, 344, 613

Gerhard, O. E. \& Binney, J. J. 1985, MNRAS, 216467

Goldstein, H. 1980, Classical Mechanics 2nd ed. (Reading: Addison-Wesley)

Goodman, J., Heggie, D. C. \& Hut, P. 1993, ApJ, 415, 715 .

Goodman, J. \& Schwarzschild, M. 1981, ApJ, 245, 1087

Guerra, D. V. \& Ratcliff, S. J. 1990, ApJ, 348, 127

Gurzadyan, V. G. \& Savvidy, G. K. 1986, A\&A, 160, 203 .

Heggie, D., 1991, in Predictability, Stability, and Chaos in N-Body Dynamical Systems, ed. A. E. Roy (Plenum Press, New York) p. 47.

Kaasalainen, M. 1994, MNRAS, 268, 1041

Kaasalainen, M., \& Binney, J.1994a, MNRAS, 268, 1033

Kaasalainen, M., \& Binney, J. 1994b, Phys. Rev. Lett., 73, 2377

Kandrup, H. E. 1998, in Long-Range Correlations in Astrophysical Systems, ed. J. R. Buchler, J. W. Dufty \& H. E. Kandrup, Ann. N Y Acad. Sci., 848, 28

Kandrup, H. E. \& Mahon, M. E. 1994, Phys. Rev. E, 49, 3735

Kormendy, J. 1985, ApJ, 292, L9

Kormendy, J. \& Richstone, D. O. 1995, ARA\&A, 33, 581

Kuo-Petravic, G., Boozer, A. H., Rome, J. A. \& Fowler, R. H. 1983, J. Comp. Phys., 51, 261

Kuzmin, G. G. 1973, in The Dynamics of Galaxies and Star Clusters, ed. G. B. Omarov (Nauka, Alma Ata)

Landau, L. D. \& Lifshitz, E. M. 1976, Mechanics 3rd ed. (Oxford: Pergamon)

Laskar, J. 1988, AAp, 198, 341

Laskar, J. 1990, Icarus, 88, 266 
Laskar, J. 1993, Physica D, 67, 257

Laskar, J. 1996, in Hamiltonian Systems with Three or More Degrees of Freedom NATO-ASI, eds. C. Simo \& A. Delshams (Dordrecht: Kluwer), REFERENCE??

Lichtenberg, A. J. \& Lieberman, M. A. 1992, Regular and Chaotic Dynamics (New York: Springer)

Lynden-Bell, D. 1967, MNRAS, 136, 101

Martens, C. C. \& Ezra, G. S. 1985, J. Chem. Phys., 83, 2990

McGill, C. A., \& Binney, J. 1990, MNRAS, 244, 634

Merritt, D. 1980, ApJS, 43, 435

Merritt, D. \& Ferrarese, L. 2001, MNRAS, 320, L30

Merritt, D. \& Fridman, T. 1996, ApJ, 460, 136

Merritt, D. \& Quinlan, G. 1998, ApJ, 498, 625

Merritt, D. \& Valluri, M. 1999, AJ, 118, 1177

Miller, R. H. 1964, ApJ, 140, 250 .

Miralda-Escudé, J. \& Schwarzschild, M. 1989, ApJ, 339, 752

Papaphilippou, Y. \& Laskar, J. 1996, A\&A, 307, 427

Papaphilippou, Y. \& Laskar, J. 1998, A\&A, 329, 451

Percival, I. C. 1974, J. Phys. A, 7, 794

Percival, I. C. 1977, J. Phys. A, 12, 57

Poincaré, H. 1892, Les Méthodes Nouvelle de la Mécanique Céleste Tome I. (Paris: GauthierVillars), ch. 3

Poon, M. \& Merritt, D. 2001, ApJ, in press (astro-ph/0006447)

Ratcliff, S. J., Chang, K. M., \& Schwarzschild, M. 1984, ApJ, 279, 610

Reiman, A. H. \& Pomphrey, N. 1991, J. Comp. Phys., 94, 225

Ryden, B. S. 1996, ApJ, 461, 146

Sambhus, N. \& Sridhar, S. 2000, Apj, 542, 143

Sanders, D. B. \& Mirabel, I. F. 1996, ARAA, 34, 749

Schechter, P. L. \& Gunn, J. E. 1979, ApJ, 229, 472

Schwarzschild, M. 1979, ApJ, 232, 236

Schwarzschild, M. 1982, ApJ, 263, 599

Shlosman, I., Begelman, M. C. \& Frank, J. 1990, Nature, 345, 679

Sridhar, S. \& Touma, J. 1997, MNRAS, 287, L1

Tremblay, B. \& Merritt, D. 1995, AJ, 110, 1039

Tremblay, B. \& Merritt, D. 1996, AJ, 111, 2243

Valluri, M. \& Merritt, D. 1998, ApJ, 506, 686

Valluri, M. \& Merritt, D. 2000, in The Chaotic Universe, ed. V. G. Gurzadyan \& R. Ruffini (Singapore: World Scientific), 229

van der Marel, R. P., Cretton, N., de Zeeuw, P. T. \& Rix, H. W. 1998, ApJ, 493, 613

Wachlin, F. C. \& Ferraz-Mello, S. 1998, MNRAS, 298, 22

Warnock, R. L. 1991, Phys. Rev. D, 66, 1803

Warnock, R. L. \& Ruth, R. D. 1991, Phys. Rev. Lett., 66, 990

Warnock, R. L. \& Ruth, R. D. 1992, Physica D, 56, 188 

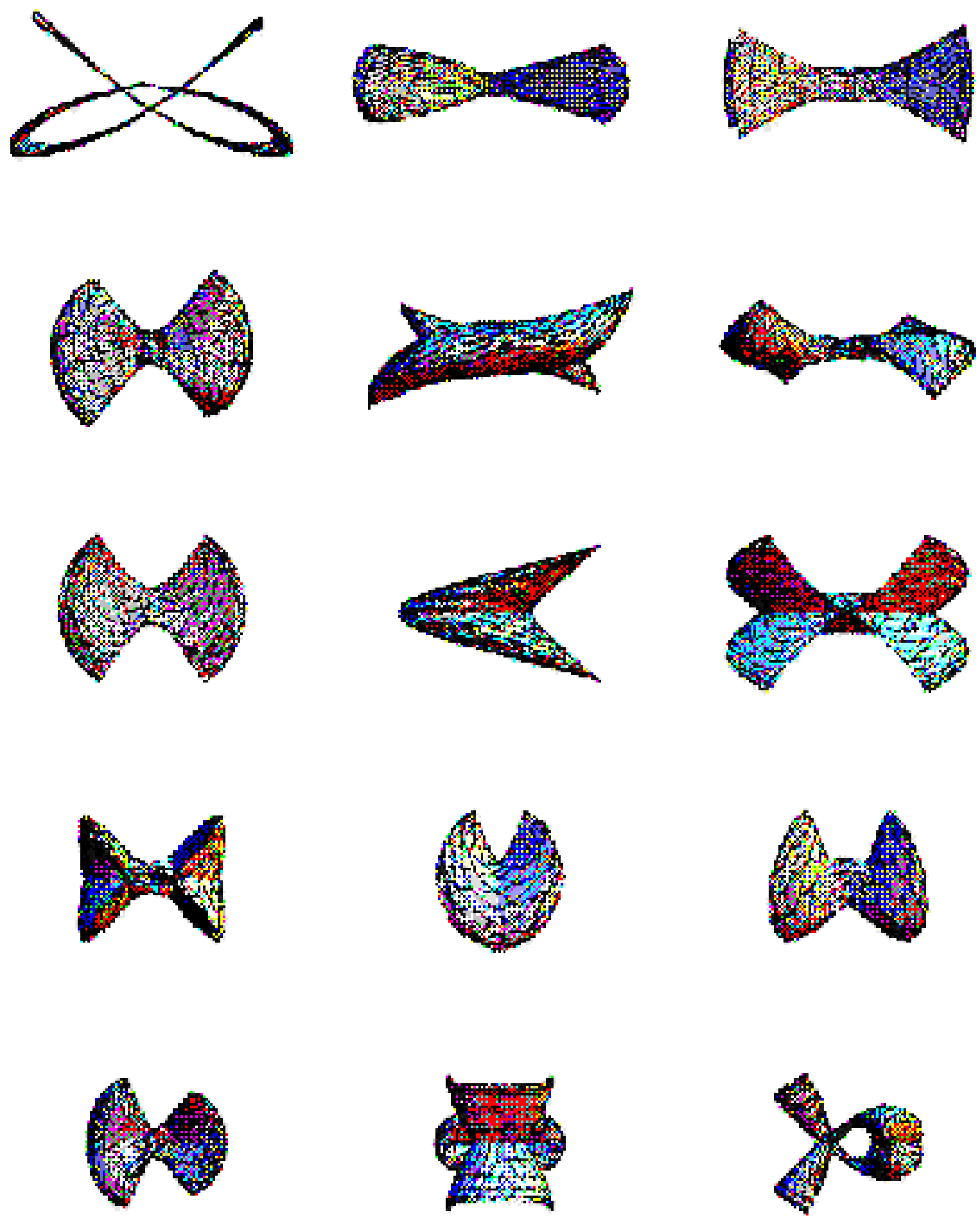

Figure 4. Surfaces filled by a set of thin, or resonant, box orbits in a non-integrable triaxial potential (Merritt $\&$ Valluri 1999), as seen from vantage points on each of the three principal axes. The cross sections of these orbits are shown in Figure 5. 
$(4,-3,0)$

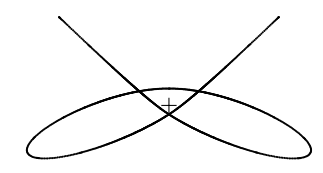

$(3,-1,-1)$

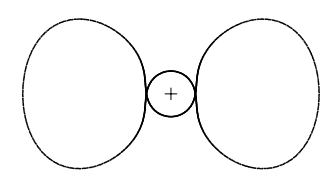

$(2,1,-2)$

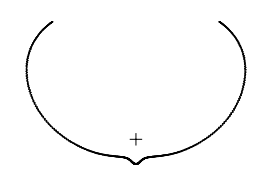

$(4,-2,-1)$

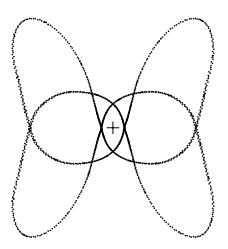

$(+)$

$(3,0,-2)$

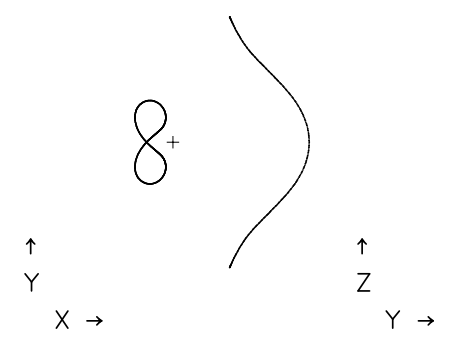

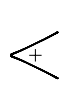
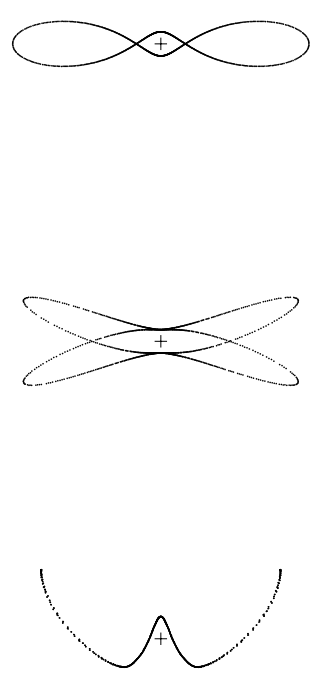

$\stackrel{+}{\infty}$

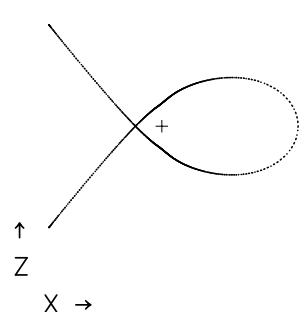

Figure 5. Intersections with the principal planes of the thin box orbits shown in Figure 4. Because the orbits are thin, their intersections with any plane define a curve or set of curves. The center of the potential is indicated by a cross. 


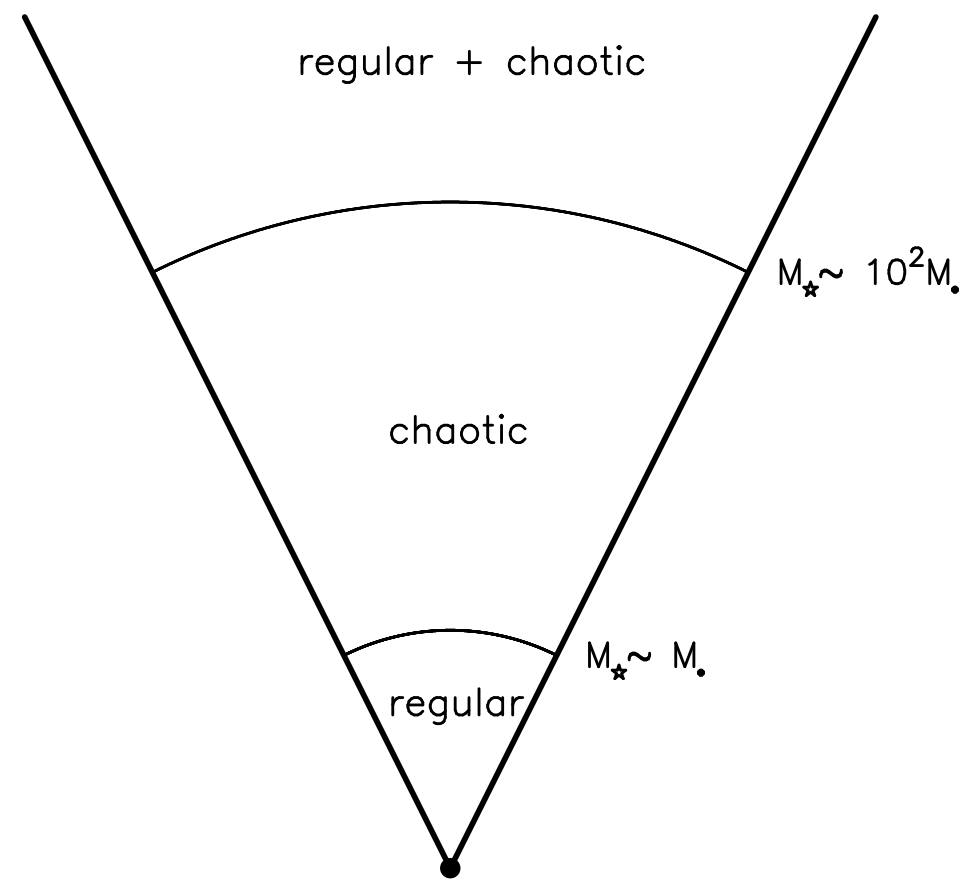

Figure 6. Three zones in the phase space of triaxial potentials (see text). 

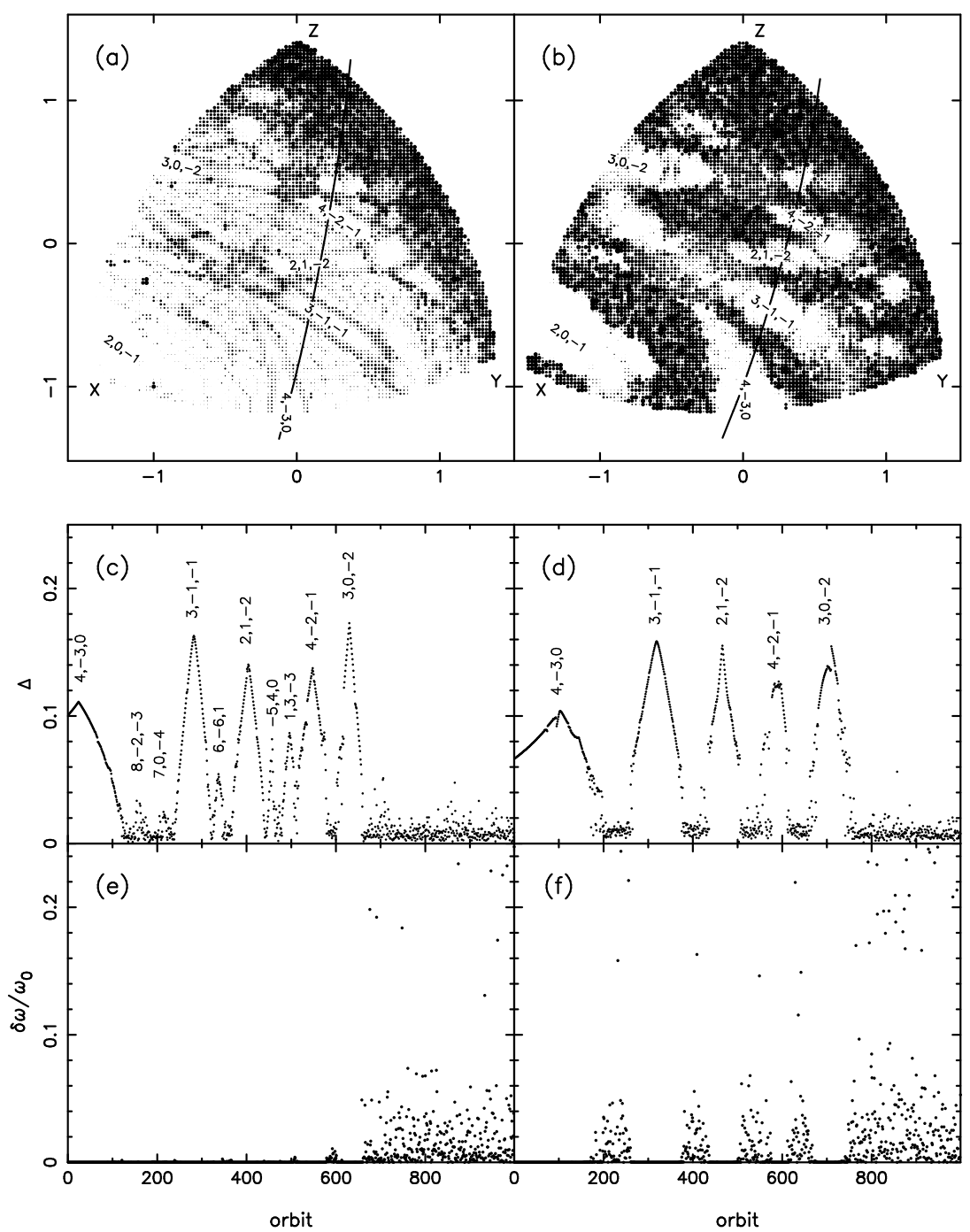

Figure 7. Non-integrability in triaxial potentials (Merritt \& Valluri 1999). The mass model in (a) has a weak $(\gamma=0.5)$ density cusp and no black hole; in (b) the black hole contains $0.3 \%$ of the total mass. Each panel shows one octant of an equipotential surface, lying close to the half-mass radius of the model; the $z$ (short) axis is vertical and the $x$ (long) axis is to the left. The grey scale measures the degree of stochasticity of orbits started with zero velocity on the equipotential surface. Stable resonance zones - the white bands in (a) and (b) - are labelled by their defining integers $\left(m_{1}, m_{2}, m_{3}\right)$. Panels (c) and (d) show the pericenter distance $\Delta$ of a set of $10^{3}$ orbits with starting points along the heavy solid lines in (a) and (b). Panels (e) and (f) plot a measure of the chaos for these orbits; $\delta \omega / \omega_{0}$ is the fractional change in the frequency of the strongest line in the orbit's frequency spectrum. 
(a)

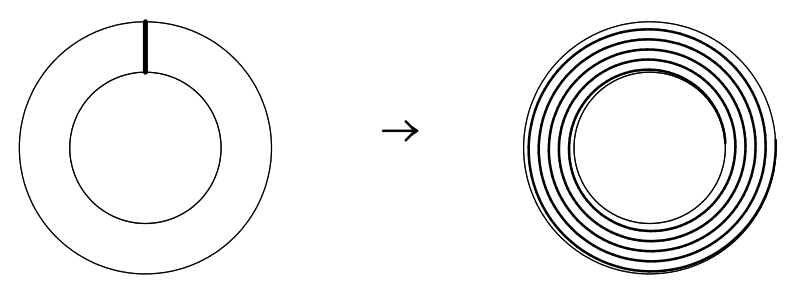

(b)

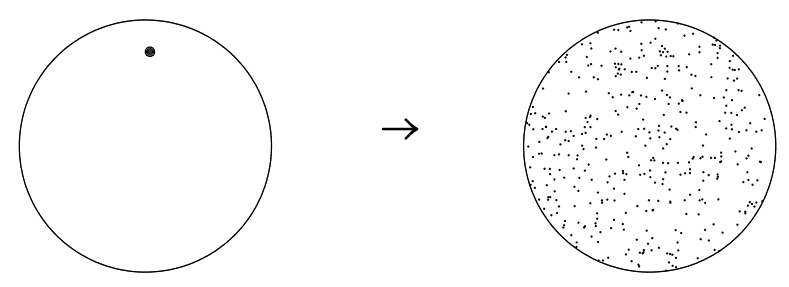

Figure 8. (a) Phase mixing vs. (b) chaotic mixing. 

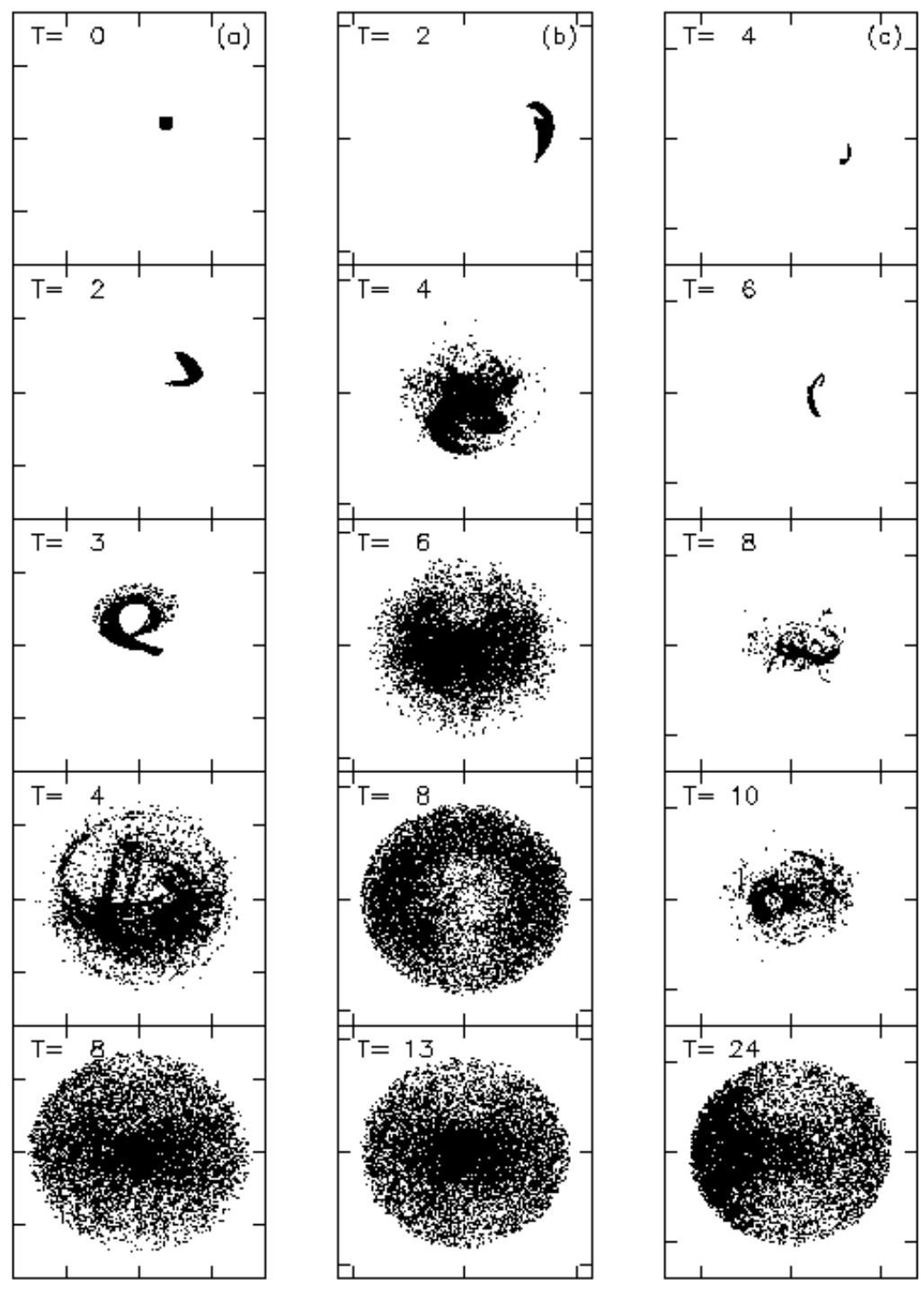

Figure 9. Mixing in a triaxial potential with a central point containing $3 \%$ of the total mass (Valluri 8 Merritt 2000). Time is in units of the local crossing time. Ensembles of $10^{4}$ phase points were distributed initially $(T=0)$ in patches on a equipotential surface with zero velocity. 

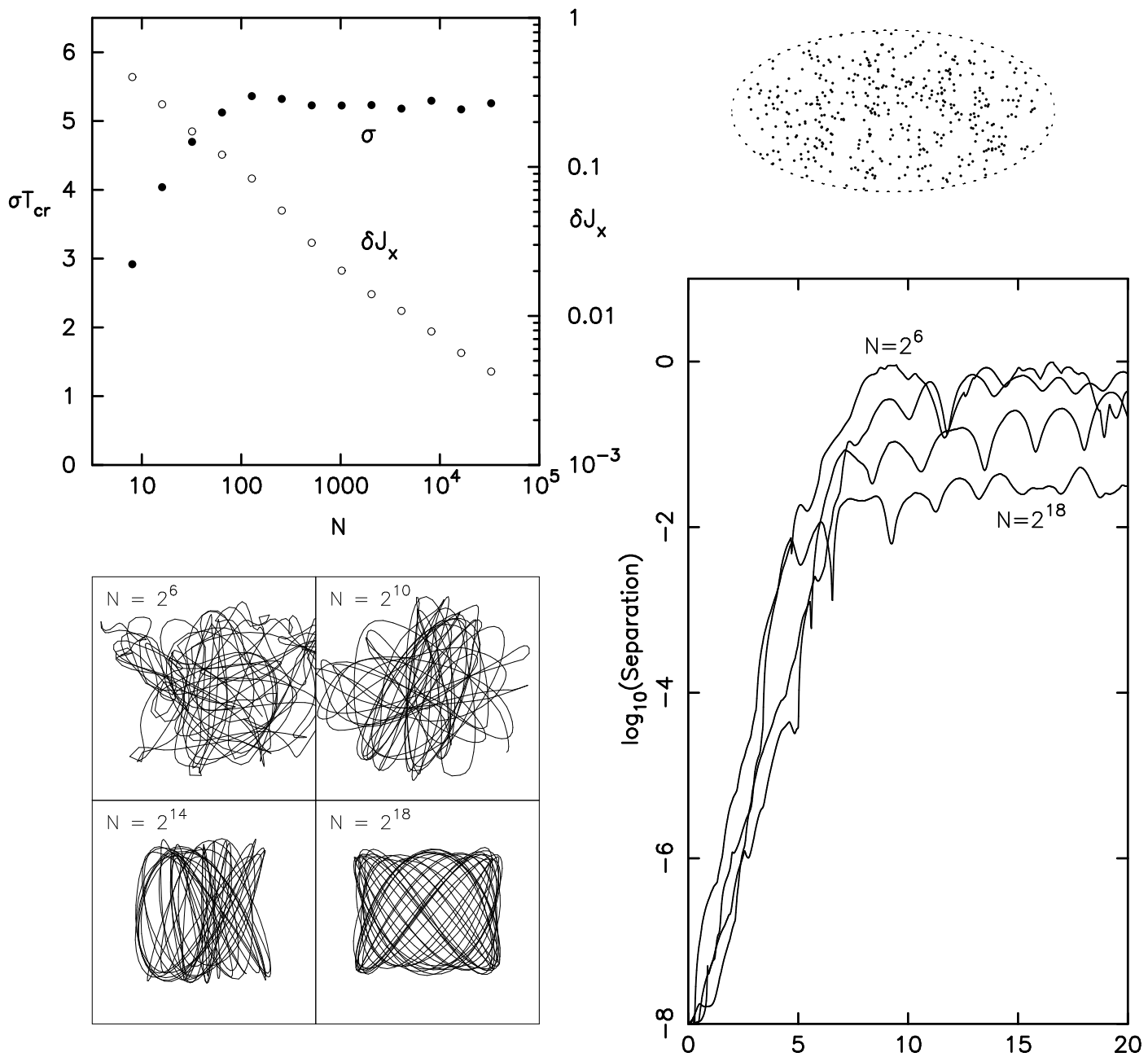

Figure 10. Evolution of orbits in a potential consisting of $\stackrel{\mathrm{t}}{N}$ fixed point masses, $m=1 / N$, distributed randomly and uniformly in an ellipsoidal volume (Valluri $\&$ Merritt 2000) (see text). 